\title{
Exosomal miRNA: an alternative mediator of cell-to-cell communication
}

\author{
Jing $\mathrm{Li}^{1 \dagger}$, Xiaohong Jiang ${ }^{1 \dagger}$ and Kehui Wang ${ }^{2^{*}}$
}

\begin{abstract}
Mounting evidence show that miRNAs are loaded into exosomes and secreted by almost all types of cells. miRNAs are selectively sorted into exosomes, and transferred to recipient cells, where they manipulate cell function. Thus, exosomal miRNAs are believed to be an alternative cell-to-cell communication mediator. Exosomal miRNAs under different pathological or physiological stimuli show different signature, indicating exosomal miRNAs are highly associated with certain diseases. Although the mechanism governing selective sorting of miRNAs are largely unclear, several mechanisms have been reviewed here. Once exosomal miRNAs released, they enter and delivery exosomal miRNAs into recipient cells, where exosomal miRNAs use cellular machinery to reduce target gene expression and manipulate cell function. Exosomal miRNAs have been proven to be implicated in the development of tumorigenesis, angiogenesis, insulin resistance and atherosclerosis. This review reveals the current understanding of exosomes miRNAs.
\end{abstract}

\section{Background}

MicroRNAs (miRNAs) are a class of small, non-coding RNAs with a length of approximately 22 nucleotides [1]. MicroRNAs play a role in vast range of physiological and pathological processes by post-transcriptionally regulating target genes [2]. Since the discovery of miRNAs in 1993, a great number of studies proved that intracellular miRNAs serve as critical mediators in metabolic disease, cardiovascular disease, development, tumour growth, and cellular stress $[1,2]$. Nevertheless, the function of miRNAs has been confined in a particular cell until the year of 2010, when extracellular microRNAs have been reported to stably exist in circulating system [3]. This discovery hugely extended the conventional view of miRNAs. Immediately after that, extracellular miRNAs were proved to be carried by exosomes, a class of $30-150 \mathrm{~nm}$ vesicles that are released from many cell types in extracellular space [4-6]. Besides, miRNAs were not randomly loaded into exosomes. Rather, with the different treatment of pathological stimuli, the profiles of exosomal miRNAs varied in content and abundance accordingly [7]. This means exosomal miRNAs are selectively and actively

\footnotetext{
* Correspondence: wangkehui@moon.ibp.ac.cn

${ }^{+}$Jing Li and Xiaohong Jiang contributed equally to this work.

${ }^{2}$ National Laboratory of Biomacromolecules, CAS Center for Excellence in

Institute of Biophysics, Chinese Academy of Sciences, Beijing 100101, China

Full list of author information is available at the end of the article
}

sorted into exosomes upon a particular treatment. Once release, exosomal miRNAs are delivered into recipient cell, where they manipulate cell function by the way the intracellular miRNAs do [7]. Given these effects, it is convincible that exosomal miRNAs are highly associated with disease and may participate the pathogenesis. Here, we aim to review the pathological profiling of exosomal miRNAs and the outcome of exosomal miRNAs communication.

\section{The release of exosomal miRNAs}

As we described above, the selective export is the most important property of exosomal miRNAs. The selective sorting is first presented by the distinctive profiling of miRNAs in exosomes and their parent cells. When analysed in exosomes derived from cells in response to the stimuli of $\mathrm{H}_{2} \mathrm{O}_{2}$, AGE and OA/PA., miRNA expressions were different. Cells exposed to AGE stimuli showed upregulation of miR-30d, miR-26b, miR-21, miR-148a, miR-24, miR-27b, and miR-27a in cells, but these miRNAs remained unchanged in exosomes upon the treatment; miR-26a, miR-29a, miR-181b, miR-150, and miR-222 were upregulated both in donor cell and exosomes; miR-25, miR-122, miR-23a, miR-103 miR-211, although upregulated in cells, were not secreted via exosomes. With the treatment of $\mathrm{H}_{2} \mathrm{O}_{2}$, miR-26b, miR-29a, and miR-222 were upregulated in cells but not released

(c) The Author(s). 2019 Open Access This article is distributed under the terms of the Creative Commons Attribution 4.0 International License (http://creativecommons.org/licenses/by/4.0/), which permits unrestricted use, distribution, and reproduction in any medium, provided you give appropriate credit to the original author(s) and the source, provide a link to the Creative Commons license, and indicate if changes were made. The Creative Commons Public Domain Dedication waiver (http://creativecommons.org/publicdomain/zero/1.0/) applies to the data made available in this article, unless otherwise stated. 
into circulating; miR-21a, miR-150, miR-181b and miR-23a, either upregulated or downregulated in cells, were upregulated into exosomes. Under high level FFAs conditions, although miR-24, miR-29a, miR-150 and miR-222 were downregulated in cell, they were increased in exosomes [7]. Another study by Goldie et al. also confirmed that the proportion of small RNAs were abundant in exosomes than that in their parent cell [8]. The selectivity of exosomal miRNAs are also proved by the miRNAs profiling varying in type and level upon different pathological stimuli. THP-1 secreted miR-30d were increased upon the treatment of AGE and OA/PA but remain unchanged upon the treatment of $\mathrm{H}_{2} \mathrm{O}_{2}$ [7]; miR-29a specially responded to the stimuli of AGE and was secreted via exosomes. These finding suggest that miRNAs are not passively released and packaged into exosomes. Quite the contrary, they are actively and selectively loaded into exosomes. Further, the profiling of secreted miRNAs varies due to the different stimuli, which are linked to a certain type of disease. Thus, this indicate that secreted miRNAs are highly associated with the pathological process.

The profiles of exosomal miRNAs are also present tissue specificity. Through comparing miRNAs profiling in exosomes among a wide range of cancer cell lines, let-7 miRNA family were found specifically increased in gastric cancer cell line, remaining unchanged in lung cancer cell line SBC-3/DMS35/NCI-H69, the colorectal cancer cell line SW480/SW620, and the stomach cancer cell line AZ-521 [9]. Besides, some miRNAs are preferentially sorted into exosomes. miR-320 family are enriched in exosomes derived from a wide range of normal or tumours cells [10-12]. miR-451 are highly expressed in exosomes derived from normal cells, such as the HMC-1 cell line, the HEK293T cell line, and EpsteinBarr virus-transformed lymphoblastic B-cell [10, 13, 14]. Microarray analysis of activation-induced miRNAs released from primary $\mathrm{T}$ lymphoblast found that miRNAs modulated upon activation are not the same in cells and exosomes. miR-575, miR-451, miR-125-3p, miR-198, miR-601 and miR887 were more highly expressed in exosomes than in cells. Conversely, some miRNAs, such as miR-17, miR-29a, let-7a, miR-142-3p, miR-181a, miR-18a, were more preferentially not loaded into exosomes [13].

Despite the specificity of exosomal miRNAs has been well established, the mechanisms governing the selective sorting of miRNAs into exosomes remained largely unclear. However, several promising mechanism has been proposed (Fig. 1). Neutral sphingomyelinase 2 (nSMase2) was believed to trigger secretion of exosomes via regulating the biosynthesis of ceramide. Reducing the activity of nSMase2 with a chemical inhibitor GW4869 or siRNA resulted in the decreased amount of miRNAs in exosomes [15]. Although this study proposed a mechanism governing miRNAs secretion, the reason for the selectivity of miRNAs secretion from different types of cell is still not quite clear. Villarroya-Beltri et al. found that miRNAs that are preferentially sorted to exosomes contain the specific short motifs (EXOmotifs). Sumoylated heterogeneous nuclear ribonucleoprotein A2B1 (hnRNPA2B1) has been demonstrated to control the loading of specific miRNAs into exosomes by binding to the motif. HnRNPA2B1 is an ubiquitous protein. According to previous reference, hnRNPA2B1 is able to bind to a RNA trafficking sequence (RTS) with a length of $21 \mathrm{nt}$ and regulate mRNA trafficking to axons in neural cell. Through comparing the RTS and the EXOmotifs identified in Villarroya-Beltri study, both two EXOmotifs are contained in this sequence. Interestingly, the hnRNPA2B1 protein in exosomes is largely sumoylated. Artificially reducing this sumoylation inhibited the binding of miRNAs to hnRNPA2B1 [15]. Interestingly, a consistent result is found in the exosome of glioblastoma multiform cells by Bolukbasi et al. They assayed the sequence of mRNAs in exosomes and found that exosomal mRNA shared a 5-nt core sequence "CTGCC" (or variations CTGC, CTCCC, CGCCC, TGCC). Moreover, miR-1289 can bind to the core sequence and mediate the mRNA secretion. A closer check of miR-1289 sequence could reveal that the EXOmotif GGAG occurred in the $5^{\prime}$-end of this miRNAs sequence [16]. This may explain why mRNAs that are able to bind to miR-1289 can be sorted into exosomes. To be specific, miR-1289 might be sorted to exosomes via sumoylated hnRNPA2B1 noted in above mentioned study, simultaneously taking complementary mRNA into exosomes. Pre-miRNA-10a, pre-miR-10b, pre-miR-21, pre-miR27a, pre-miR-155, and pre-miR-373, along with RISC-loading complex (RLC) which consists of Dicer, AGO2, and TRBP, are present in exosomes of cancer cells [17]. These pre-miRNAs can be processed into mature miRNAs by RLC in exosomes [17-19]. This study provide a new mechanism of exosomal miRNA sorting.

\section{The uptake of exosomal miRNA by recipient cells}

Once released, exosomal miRNAs, after circulating in body fluids for a short period of time, will be delivered to recipient cells, where they appear to use a range of mechanisms to bind on target cell and undergo internalization [20, 21]. Surface protein on exosomes facilitate exosomes first adhere to target cell, which is thought to be a fundamental step for exosomes-target cell communication $[22,23]$. Tetrapanins are thought to have a role in adhesion, motility, signal transduction and cell activation, and they are highly abundant on the exosomes surface [24-26]. These tetrapanins include CD9, CD53, CD63, CD81 and CD82, which may contribute to the spatial assemble for antigen recognition and may partially dictate the signal induced by the exosomes [26-31]. 

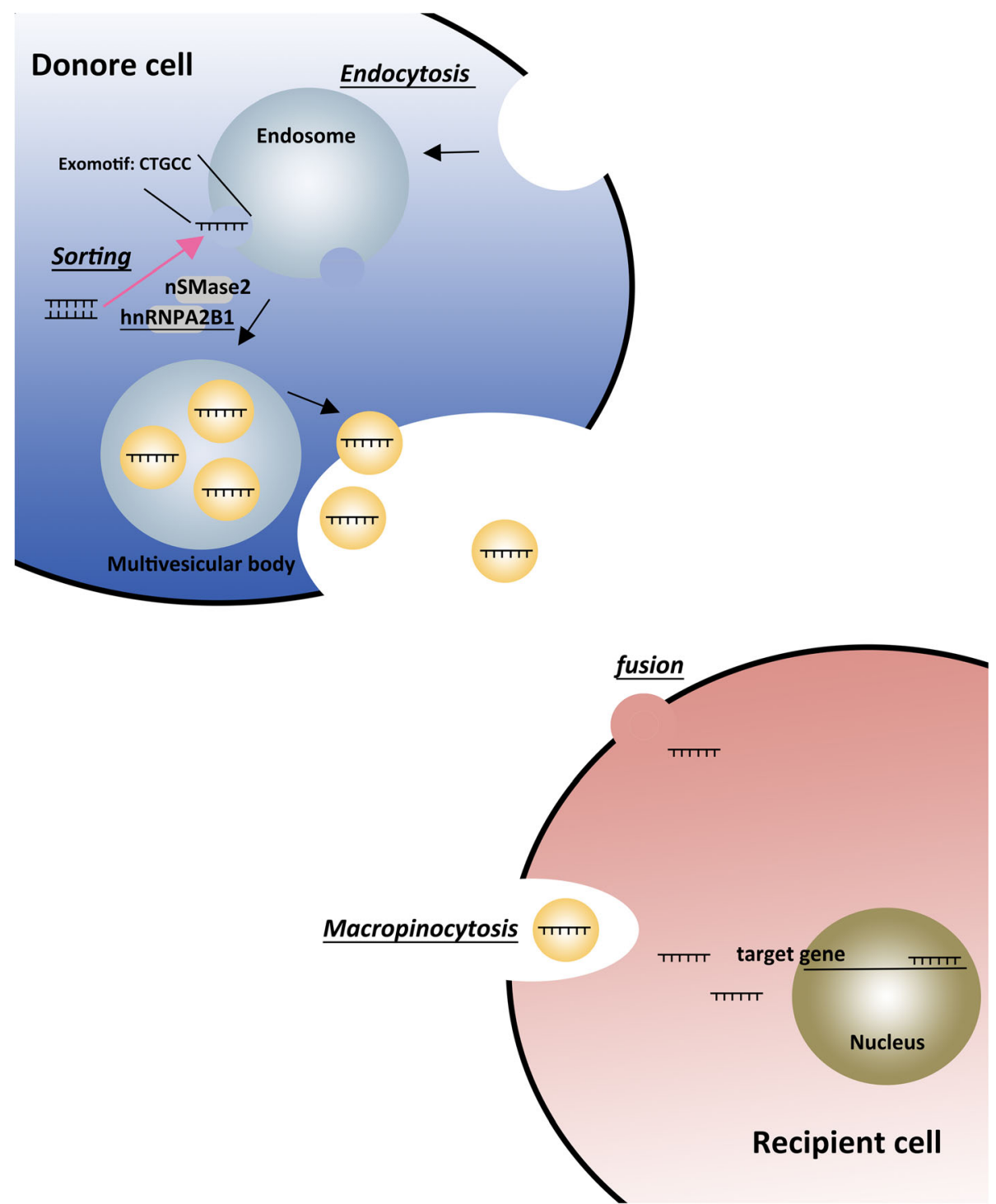

Fig. 1 The machanism of extracellular release and uptake

Treatment of antibodies on recipient cells against the tetraspanins CD81 or CD9 can reduce the uptake of exosomes by dentritic cells, which suggest that tetraspanins have a role in exosomes uptaking and function [32, 33]. Intergrin is another type of adhesion protein playing a role in exosomes binding. The treatment of antibodies against integrins $\alpha \mathrm{v}$ (CD51) and $\beta 3$ (CD61) on the dentritic cell surface reduce the uptake of exosomes [32]. When adhering, intergrins change to a high affinity status and assist the high avidity binding of cell to the integrin-bound cell $[34,35]$. Inducing a high-affinity state of LFA-1 by manganese chloride on resting T-cells cause a significant increase of exosomes uptake [35, 36]; conversely, using an antagonistic antibody to hinder the formation of high-affinity state inhibit this process [37].
Internalization is the next step in exosomes' destiny, and also the fundamental process for exosomes delivering miRNAs into target cell and cellular response [5, 38-40]. The capacity of cells up-taking exosomes dramatically reduced at $4 \mathrm{C}$, which suggest that exosome uptake is an energy-dependent process [41]. The mechanism underlying internalization of exosomes are still being subject to debate. Nevertheless, there are four pathways have been implicated in exsomes uptake, including: fusion of the exosome membrane with the plasma membrane [42, 43]; Phagocytosis [44] and micropinocytosis; macropinocytosis [45, 46]; Clathrin-mediated endocytosis (CME) [47-49]; and Caveolin-dependent endocytosis [50-53], and lipid raft-mediated endocytosis [54-56]. 
The cellular response of exosomal miRNAs in recipient cells Exosomal miRNAs can cause a range of response in recipient cell, where they are internalized and play function through post-transcriptionally silencing target gene $[7,57]$. Exosomal miRNAs has been widely implicated in many steps of tumorigenesis. Zhang et al. discovered that exosomal monocytic miR-150 released by AGEtreated THP-1 serving as inflammatory factor can enter endothelial cells and enhance endothelial cell migration [7]. Endothelial cell migration is one of the important step of angiogenesis, which play a role in tumorigenesis. The following study continued to demonstrate that exosomal miR-150 from monocyte increase the angiogenesis by targeting c-Myc, which in turn result in enhanced tumour growth [58]. Further study demonstrated exosomal miR-150 from monocyte increase VEGF secretion of tumour-associated macrophage, which induce angiogenesis by recruiting endothelial cell [59]. The series of research demonstrate that, under chronic inflammation condition, THP-1 derived exosomal miR-150 contribute to tumorigenesis. miR-223, a tumour-associated macrophage (TAM) specific miRNA, can be released and transferred to breast cancer cell, where they activate the Mef2c-b-catenin pathway and enhance the invasiveness [60]. Tumours also are able to release miRNAs to manipulate microenvironment and facilitate the growth of themselves. MiR-214, which is thought to be onco-miRNAs has been reported to be released by tumour cells and travel to $\mathrm{T}$ regular cell. They reduce the expression of PTEN and activate the $\mathrm{T}$ regular cell, which in turn promote the immune escape [57]. Exosomal miRNAs also have a role in tumour metastasis outgrowth. In tumour cells disseminating to brain, the expression level of PTEN specifically reduced compared to primary tumour or other organ metastases. Further investigation found that astrocyte-derived exosomal miR-19a down-regulate PTEN expression in metastatic tumour cells, which in turn cause CCL2 up-regulation and enhance the outgrowth of brain metastatic tumour cells [61]. Another study also demonstrated exosomal miRNAs enhance the metastasis of cancer cell. Breast cancer cell lines MCF-10A and MDA-MB-231 released miR-105 reduced ZO-1 gene expression in endothelial cells and enhance the metastases to the lung and brain [62].

Exosomal miRNAs are also implicated metabolic diseases including, insulin resistance, and cardiovascular disease. Adipose tissue, a major organ for energy balancing, has been demonstrated by a number of studies that it can release considerable amount of exosomal miRNAs which manipulate metabolism [63]. Recent study demonstrate that adipose tissue secreted not only adipokines, but also exosomal miRNAs [64]. Specifically impairing the maturation of miRNAs in adipose tissue by knocking out the miRNAs-processing enzyme Dicer results in a substantial decrease in levels of exosomal miRNAs in circulating. In parallel experiments, the transplantation of white and brown adipose tissue into $\mathrm{KO}$ mice restore the level of insulin resistance [64]. This study proposed a conclusion that adipose tissue is a major source of circulating exosomal miRNAs. Further investigation into the

Table 1 The type of exosomal miRNAs and their involvements in the pathogenesis of diseases

\begin{tabular}{|c|c|c|c|c|}
\hline Names of miRNA & Donor cell & Recipient cell & $\begin{array}{l}\text { intercellular Target gene } \\
\text { and function }\end{array}$ & Ref. \\
\hline miR-150 & Monocyte (THP-1) & Endothelial cell & c-myc, promoting angiogenesis & {$[7,58,59]$} \\
\hline miR-19a & Brain astrocyte & $\begin{array}{l}\text { Tumour cells metastasis } \\
\text { to brain }\end{array}$ & $\begin{array}{l}\text { PTEN, prime brain metastasis } \\
\text { outgrowth }\end{array}$ & [61] \\
\hline miR-214 & Tumour cell & Regulatory T cell & PTEN, induce immune escape & [57] \\
\hline miR-155 & Adipose tissue macrophage (obese) & $\begin{array}{l}\text { Liver, Muscle, adipose } \\
\text { tissue }\end{array}$ & PPARY & [65] \\
\hline miR-223 & tumour-associated macrophage & Tumour cells (breast cancer) & $\begin{array}{l}\text { Activate Mef2c-b-catenin } \\
\text { pathway }\end{array}$ & {$[60]$} \\
\hline miR-105 & Breast cancer & Endothelial cell & $\begin{array}{l}\text { ZO- } 1 \text {, promote the metastasis } \\
\text { to brain and lung }\end{array}$ & [62] \\
\hline miR-143/miR-145 & Endohelial cells & Vascular smooth muscle & Atheroprotective role & {$[67,68]$} \\
\hline miR-26a, miR-29a, miR-181b, miR-222 & Monocyte (THP-1) exposed to AGE & - & - & [7] \\
\hline miR-21, miR-150, miR-181b, miR-23a & Monocyte (THP-1) exposed to $\mathrm{H}_{2} \mathrm{O}_{2}$ & - & - & [7] \\
\hline miR-29a, miR-24a, miR-222 & Monocyte (THP-1) & - & - & [7] \\
\hline miR-451 & $\begin{array}{l}\text { HMC-1 HEK293T lymphoblastic } \\
\text { B-cell }\end{array}$ & - & - & {$[10,13,14]$} \\
\hline miR-320 & Normal cell and tumour cells & - & - & [10-12] \\
\hline $\begin{array}{l}\text { miR-575, miR-451, miR-125-3p, } \\
\text { miR-198, miR-601, miR887 }\end{array}$ & primary T lymphoblast & - & - & [13] \\
\hline
\end{tabular}


exosomal miRNAs found that adipose tissue macrophage release miR-155, which enter liver, muscle and adipocyte and modulate insulin sensitivity [65]. Large adipocytes transfer to small adipocytes, which in turn induce lipid storage [66]. Exosomal miRNAs also involved in the development of atherosclerosis [67]. A study found that endothelial cells also are able to secrete exosomal miR-143 and miR-145, which then alter the phenotype of vascular smooth muscle, playing an atheroprotective role in the development of sclerosis [68].

\section{Conclusion}

As the study of exosomal miRNAs mounting recent years, a novel miRNA-based communication network is forming in several defined diseases. Exosomal miRNAs, which are selectively and actively loaded into exosomes, are highly implicated in many pathogenesis process (Table 1). Despite not investigated comprehensively, exosomal miRNAs are considered as an alternative cell-tocell communication mediator, which may play a role in pathogenesis. Further investigating the function of exosomal miRNAs can lead to the brand new understanding of many defined diseases. Nevertheless, due to the finite study strategy, the secretion of exosomal in health and disease in vivo is rarely understood. It is easy to observe the communication of exosomal miRNAs in vitro, but it is hard to grab the direct evidence of the transfer of exosomal miRNAs in vivo. The new research strategy and new cutting-edge technologies should be improved and utilized in this filed. More deep investigation into exsomal miRNAs physiological and pathological function in vivo should be performed.

\section{Abbreviations \\ CME: Clathrin-mediated endocytosis; hnRNPA2b1: heterogenous nuclear ribonucleoprotein A2B1; LFA-1: Lymphocyte function associated antigen-1; miRNAs: microRNA; mRNA: messenger RNA; nSMase2: sphingomyelinase 2; OA: Oleic acid; PA: Palmitic acid; pre-miRNA: precursor microRNA; RLC: RISC- loading complex; TAM: Tumour-associated macrophage}

\section{Acknowledgments}

Not applicable

\section{Funding}

This work was supported by the National Natural Science Foundation of China. (Grant No. 31741066), the Fundamental Research Funds for the Central Universities. (Grant No. 020814370087, 020814370094).

\section{Availability of data and materials}

$$
\text { Not applicable }
$$

\section{Authors' contributions}

$J \mathrm{~L}$ and $\mathrm{XJ}$ conducted the literature search and drafted the manuscript. KW revised the text to produce the final version of the manuscript. All authors read and approved the final manuscript.

\section{Ethics approval and consent to participate}

Not applicable

\section{Consent for publication}

Not applicable

\section{Competing interests}

The authors declare that they have no competing interests.

\section{Publisher's Note}

Springer Nature remains neutral with regard to jurisdictional claims in published maps and institutional affiliations.

\section{Author details}

${ }^{1}$ State Key Laboratory of Pharmaceutical Biotechnology, Collaborative Innovation Centre of Chemistry for Life Sciences, Jiangsu Engineering Research Centre for MicroRNA Biology and Biotechnology, NJU Advanced Institute for Life Sciences (NAILS), School of life sciences, Nanjing University, 163 Xianlin Road, Nanjing 210023, Jiangsu, China. ${ }^{2}$ National Laboratory of Biomacromolecules, CAS Center for Excellence in Institute of Biophysics, Chinese Academy of Sciences, Beijing 100101, China.

Received: 20 July 2018 Accepted: 15 March 2019

Published online: 11 July 2019

\section{References}

1. Ambros $V$. The functions of animal microRNAs. Nature. 2004;431:350-5. https://doi.org/10.1038/nature02871.

2. Bartel DP. MicroRNAs: genomics, biogenesis, mechanism, and function. Cell. 2004;116:281-97.

3. Chen $X$, et al. Characterization of microRNAs in serum: a novel class of biomarkers for diagnosis of cancer and other diseases. Cell Res. 2008;18: 997-1006. https://doi.org/10.1038/cr.2008.282.

4. Thery C, Zitvogel L, Amigorena S. Exosomes: composition, biogenesis and function. Nat Rev Immunol. 2002;2:569-79. https://doi.org/10.1038/nri855.

5. Valadi $\mathrm{H}$, et al. Exosome-mediated transfer of mRNAs and microRNAs is a novel mechanism of genetic exchange between cells. Nat Cell Biol. 2007;9: 654-9. https://doi.org/10.1038/ncb1596.

6. Chen $\mathrm{X}$, Liang $\mathrm{H}$, Zhang J, Zen $\mathrm{K}$, Zhang $\mathrm{CY}$. Horizontal transfer of microRNAs: molecular mechanisms and clinical applications. Protein Cell. 2012;3:28-37. https://doi.org/10.1007/s13238-012-2003-z.

7. Zhang $Y$, et al. Secreted monocytic miR-150 enhances targeted endothelial cell migration. Mol Cell. 2010;39:133-44. https://doi.org/10.1016/j.molcel. 2010.06.010

8. Goldie BJ, et al. Activity-associated miRNA are packaged in Map1b-enriched exosomes released from depolarized neurons. Nucleic Acids Res. 2014;42: 9195-208. https://doi.org/10.1093/nar/gku594.

9. Ohshima $\mathrm{K}$, et al. Let-7 microRNA family is selectively secreted into the extracellular environment via exosomes in a metastatic gastric cancer cell line. PLoS One. 2010;5:e13247. https://doi.org/10.1371/journal.pone.0013247.

10. Guduric-Fuchs J, et al. Selective extracellular vesicle-mediated export of an overlapping set of microRNAs from multiple cell types. BMC Genomics. 2012;13:357. https://doi.org/10.1186/1471-2164-13-357.

11. Squadrito $M L$, et al. Endogenous RNAs modulate microRNA sorting to exosomes and transfer to acceptor cells. Cell Rep. 2014;8:1432-46. https://doi.org/10.1016/j.celrep.2014.07.035

12. Liao J, Liu R, Yin L, Pu Y. Expression profiling of exosomal miRNAs derived from human esophageal cancer cells by Solexa high-throughput sequencing. Int J Mol Sci. 2014;15:15530-51. https://doi.org/10.3390/ijms150915530.

13. Villarroya-Beltri $C$, et al. Sumoylated hnRNPA2B1 controls the sorting of miRNAs into exosomes through binding to specific motifs. Nat Commun. 2013;4:2980. https://doi.org/10.1038/ncomms3980.

14. Koppers-Lalic D, et al. Nontemplated nucleotide additions distinguish the small RNA composition in cells from exosomes. Cell Rep. 2014;8:1649-58. https://doi.org/10.1016/j.celrep.2014.08.027.

15. Kosaka N, et al. Neutral sphingomyelinase 2 (nSMase2)-dependent exosomal transfer of angiogenic microRNAs regulate cancer cell metastasis. J Biol Chem. 2013;288:10849-59. https://doi.org/10.1074/jbc.M112.446831.

16. Bolukbasi MF, et al. miR-1289 and "Zipcode"-like sequence enrich mRNAs in microvesicles. Molecular therapy Nucleic acids. 2012;1:e10. https://doi.org/ 10.1038/mtna.2011.2.

17. Melo SA, et al. Cancer exosomes perform cell-independent microRNA biogenesis and promote tumorigenesis. Cancer Cell. 2014;26:707-21. https://doi.org/10.1016/j.ccell.2014.09.005.

18. Chendrimada TP, et al. TRBP recruits the dicer complex to Ago2 for microRNA processing and gene silencing. Nature. 2005:436:740-4. https://doi.org/10.1038/nature03868. 
19. Gregory RI, Chendrimada TP, Cooch N, Shiekhattar R. Human RISC couples microRNA biogenesis and posttranscriptional gene silencing. Cell. 2005;123: 631-40. https://doi.org/10.1016/j.cell.2005.10.022.

20. Saunderson SC, Dunn AC, Crocker PR, McLellan AD. CD169 mediates the capture of exosomes in spleen and lymph node. Blood. 2014;123:208-16. https://doi.org/10.1182/blood-2013-03-489732.

21. Huang-Doran I, Zhang CY, Vidal-Puig A. Extracellular vesicles: novel mediators of cell communication in metabolic disease. Trends Endocrinol Metab. 2017;28: 3-18. https://doi.org/10.1016/j.tem.2016.10.003.

22. McKelvey KJ, Powell KL, Ashton AW, Morris JM, McCracken SA. Exosomes: mechanisms of uptake. Journal of circulating biomarkers. 2015;4:7. https:// doi.org/10.5772/61186

23. Muller $L$, et al. Human tumor-derived exosomes (TEX) regulate Treg functions via cell surface signaling rather than uptake mechanisms. Oncoimmunology. 2017;6:e1261243. https://doi.org/10.1080/2162402X. 2016.1261243

24. Hemler ME. Tetraspanin functions and associated microdomains. Nat Rev Mol Cell Biol. 2005;6:801-11. https://doi.org/10.1038/nrm1736.

25. Zoller M. Tetraspanins: push and pull in suppressing and promoting metastasis. Nat Rev Cancer. 2009;9:40-55. https://doi.org/10.1038/nrc2543.

26. Escola JM, et al. Selective enrichment of tetraspan proteins on the internal vesicles of multivesicular endosomes and on exosomes secreted by human B-lymphocytes. J Biol Chem. 1998;273:20121-7.

27. Thery C, et al. Molecular characterization of dendritic cell-derived exosomes. Selective accumulation of the heat shock protein hsc73. J Cell Biol. 1999; 147:599-610.

28. Heijnen HF, Schiel AE, Fijnheer R, Geuze HJ, Sixma JJ. Activated platelets release two types of membrane vesicles: microvesicles by surface shedding and exosomes derived from exocytosis of multivesicular bodies and alphagranules. Blood. 1999;94:3791-9.

29. Rubinstein $E$, et al. Reduced fertility of female mice lacking CD81. Dev Biol. 2006;290:351-8. https://doi.org/10.1016/j.ydbio.2005.11.031.

30. Zhu GZ, et al. Residues SFQ (173-175) in the large extracellular loop of CD9 are required for gamete fusion. Development. 2002;129:1995-2002.

31. Helming L, Gordon S. The molecular basis of macrophage fusion. Immunobiology. 2007;212:785-93. https://doi.org/10.1016/j.imbio.2007.09.012

32. Morelli $A E$, et al. Endocytosis, intracellular sorting, and processing of exosomes by dendritic cells. Blood. 2004;104:3257-66. https://doi.org/10. 1182/blood-2004-03-0824.

33. Nazarenko I, et al. Cell surface tetraspanin Tspan 8 contributes to molecular pathways of exosome-induced endothelial cell activation. Cancer Res. 2010; 70:1668-78. https://doi.org/10.1158/0008-5472.CAN-09-2470.

34. Cano A, et al. Expression pattern of the cell adhesion molecules. E-cadherin, P-cadherin and alpha 6 beta 4 intergrin is altered in pre-malignant skin tumors of p53-deficient mice. Int J Cancer. 1996;65:254-62. https://doi.org/ 10.1002/(SICI)1097-0215(19960117)65:2<254::AID-IJC21>3.0.CO;2-C.

35. Nolte-'t Hoen EN, Buschow SI, Anderton SM, Stoorvogel W, Wauben MH. Activated T cells recruit exosomes secreted by dendritic cells via LFA-1. Blood. 2009;113:1977-81. https://doi.org/10.1182/blood-2008-08-174094.

36. Hao $\mathrm{S}$, et al. Mature dendritic cells pulsed with exosomes stimulate efficient cytotoxic T-lymphocyte responses and antitumour immunity. Immunology. 2007;120:90-102. https://doi.org/10.1111/j.1365-2567.2006.02483.x.

37. Nanjundappa RH, et al. GP120-specific exosome-targeted T cell-based vaccine capable of stimulating DC- and CD4(+) T-independent CTL responses. Vaccine. 2011;29:3538-47. https://doi.org/10.1016/j.vaccine.2011.02.095.

38. Al-Mayah AH, Irons SL, Pink RC, Carter DR, Kadhim MA. Possible role of exosomes containing RNA in mediating nontargeted effect of ionizing radiation. Radiat Res. 2012;177:539-45.

39. Alvarez-Erviti L, et al. Delivery of siRNA to the mouse brain by systemic injection of targeted exosomes. Nat Biotechnol. 2011;29:341-5. https://doi. org/10.1038/nbt.1807.

40. Montecalvo A, et al. Mechanism of transfer of functional microRNAs between mouse dendritic cells via exosomes. Blood. 2012;119:756-66. https://doi.org/10.1182/blood-2011-02-338004.

41. Mulcahy LA, Pink RC, Carter DR. Routes and mechanisms of extracellular vesicle uptake. Journal of extracellular vesicles. 2014;3. https://doi.org/10 3402/jev.v3.24641.

42. Del Conde I, Shrimpton CN, Thiagarajan P, Lopez JA. Tissue-factor-bearing microvesicles arise from lipid rafts and fuse with activated platelets to initiate coagulation. Blood. 2005:106:1604-11. https://doi.org/10.1182/ blood-2004-03-1095.
43. Parolini l, et al. Microenvironmental $\mathrm{pH}$ is a key factor for exosome traffic in tumor cells. J Biol Chem. 2009;284:34211-22. https://doi.org/10.1074/jbc.M109.041152.

44. Feng $\mathrm{D}$, et al. Cellular internalization of exosomes occurs through phagocytosis. Traffic. 2010;11:675-87. https:/doi.org/10.1111/j.1600-0854.2010.01041.x.

45. Doherty GJ, McMahon HT. Mechanisms of endocytosis. Annu Rev Biochem. 2009;78:857-902. https://doi.org/10.1146/annurev.biochem.78.081307.110540.

46. Swanson JA. Shaping cups into phagosomes and macropinosomes. Nat Rev Mol Cell Biol. 2008;9:639-49. https://doi.org/10.1038/nrm2447.

47. Kirchhausen T. Clathrin. Annu Rev Biochem. 2000;69:699-727. https://doi. org/10.1146/annurev.biochem.69.1.699.

48. Wang LH, Rothberg KG, Anderson RG. Mis-assembly of clathrin lattices on endosomes reveals a regulatory switch for coated pit formation. J Cell Biol. 1993;123:1107-17.

49. Ehrlich $M$, et al. Endocytosis by random initiation and stabilization of clathrin-coated pits. Cell. 2004;118:591-605. https://doi.org/10.1016/j.cell. 2004.08.017.

50. Anderson RG. The caveolae membrane system. Annu Rev Biochem. 1998;67: 199-225. https://doi.org/10.1146/annurev.biochem.67.1.199.

51. Kurzchalia TV, Parton RG. Membrane microdomains and caveolae. Curr Opin Cell Biol. 1999:11:424-31.

52. Lavie $Y$, Fiucci G, Czarny M, Liscovitch M. Changes in membrane microdomains and caveolae constituents in multidrug-resistant cancer cells. Lipids. 1999; 34(Suppl):S57-63.

53. Patel HH, Murray F, Insel PA. G-protein-coupled receptor-signaling components in membrane raft and caveolae microdomains. Handb Exp Pharmacol. 2008: 167-84. https://doi.org/10.1007/978-3-540-72843-6 7.

54. Riemann D, et al. Caveolae/lipid rafts in fibroblast-like synoviocytes: ectopeptidase-rich membrane microdomains. Biochem J. 2001;354:47-55.

55. Schroeder $\mathrm{F}$, et al. Recent advances in membrane microdomains: rafts, caveolae, and intracellular cholesterol trafficking. Exp Biol Med (Maywood). 2001;226:873-90.

56. Badizadegan $\mathrm{K}$, et al. Floating cholera toxin into epithelial cells: functional association with caveolae-like detergent-insoluble membrane microdomains. Int J Med Microbiol. 2000;290:403-8. https://doi.org/10.1016/S14384221(00)80052-1.

57. Yin $Y$, et al. Tumor-secreted miR-214 induces regulatory $T$ cells: a major link between immune evasion and tumor growth. Cell Res. 2014;24:1164-80. https://doi.org/10.1038/cr.2014.121.

58. Li J, et al. Microvesicle-mediated transfer of microRNA-150 from monocytes to endothelial cells promotes angiogenesis. J Biol Chem. 2013;288:23586-96. https://doi.org/10.1074/jbc.M113.489302.

59. Liu Y, et al. Microvesicle-delivery miR-150 promotes tumorigenesis by up-regulating VEGF, and the neutralization of miR-150 attenuate tumor development. Protein Cell. 2013;4:932-41. https://doi.org/10.1007/ s13238-013-3092-z.

60. Yang $M$, et al. Microvesicles secreted by macrophages shuttle invasionpotentiating microRNAs into breast cancer cells. Mol Cancer. 2011;10: 117. https://doi.org/10.1186/1476-4598-10-117.

61. Zhang $L$, et al. Microenvironment-induced PTEN loss by exosomal microRNA primes brain metastasis outgrowth. Nature. 2015;527:100-4. https://doi.org/10.1038/nature15376.

62. Zhou W, et al. Cancer-secreted miR-105 destroys vascular endothelial barriers to promote metastasis. Cancer Cell. 2014;25:501-15. https://doi.org/10.1016/j. ccr.2014.03.007.

63. Ogawa R, et al. Adipocyte-derived microvesicles contain RNA that is transported into macrophages and might be secreted into blood circulation. Biochem Biophys Res Commun. 2010;398:723-9. https://doi.org/10.1016/j.bbrc.2010.07.008.

64. Thomou T, et al. Adipose-derived circulating miRNAs regulate gene expression in other tissues. Nature. 2017;542:450-5. https://doi.org/10.1038/nature21365.

65. Ying W, et al. Adipose tissue macrophage-derived Exosomal miRNAs can modulate in vivo and in vitro insulin sensitivity. Cell. 2017;171:372-384 e312. https://doi.org/10.1016/j.cell.2017.08.035.

66. Muller G, Schneider M, Biemer-Daub G, Wied S. Microvesicles released from rat adipocytes and harboring glycosylphosphatidylinositol-anchored proteins transfer RNA stimulating lipid synthesis. Cell Signal. 2011;23:1207-23. https:// doi.org/10.1016/j.cellsig.2011.03.013.

67. Rader DJ, Parmacek MS. Secreted miRNAs suppress atherogenesis. Nat Cell Biol. 2012;14:233-5. https://doi.org/10.1038/ncb2452.

68. Hergenreider $\mathrm{E}$, et al. Atheroprotective communication between endothelial cells and smooth muscle cells through miRNAs. Nat Cell Biol. 2012;14:249-56. https://doi.org/10.1038/ncb2441. 